entwickelt werden, nicht um daraus einen neuen Ansatz zu machen, sondern um das reiche Spektrum der Praxis mit einem solchen Modell besser nutzen zu können.

Im ersten Kapitel führt der Autor in die Grundfragen der Personzentrierten Systemtheorie ein und erläutert die vier Verstehensperspektiven mit dem individualistischpsychodynamisch-humanistischen, dem interpersonellsystemdynamischen, dem organismisch-körperlichen und dem gesellschaftlich-kulturellen Fokus. Er illustriert diese theoretischen Ausführungen mit vier Fallvignetten.

Im zweiten Kapitel folgen einleitend Überlegungen zum Begriff System, um dann die Themen «Subjektivität» und «Objektivität» mit Bezügen zur Philosophie zu vertiefen. Das Kapitel steht unter der Überschrift «Leben als Zeichenprozess - die Perspektive der Biosemiotik». Die Biosemiotik legt eine untrennbare Verwobenheit von psychischen und interpersonellen Phänomenen mit körperlich-evolutionären sowie kulturell-makrosozialen Prozessen nahe. Spannend sind seine Ausführungen zu den Grenzen der Subjektivität, der Bedeutungszuschreibung zu Phänomenen mit Bezügen zur Phänomenologie, der Gestaltpsychologie als Wahrnehmungstheorie und der Experimentellen Psychologie. Auch diese Ausführungen erhalten mit einer Fallvignette einen Praxisbezug.

Im dritten Kapitel werden (essenzielle) Prinzipien der Systemtheorie erläutert. Es geht immer um Prozesse von miteinander dynamisch vernetzten «Teilen». Die Gesamtheit wechselseitig voneinander abhängiger Einflüsse wird als Feld betrachtet. Zu diesen Prinzipien zählt zum Beispiel die Rückkoppelung: Die Veränderung bei einem Teil pflanzt sich im System fort. Oder die Mikro-/Makro-Ebene: Die vernetze und rückgekoppelte Dynamik der «Teile» bildet bottom-up "Ordnungen» aus, die dann top-down die weitere Dynamik der «Teile» bestimmen. Diese Ordnungen sind selbstorganisiert. Oder System versus Umgebung: Selbstorganisation ist zwar etwas dem System Inhärentes, aber immer auch mit Adaptionen an die Systemumgebungen verbunden. Diese Essentials werden in Unterkapiteln wissenschaftstheoretisch mit Bezug zu Forschung und Praxis vertieft. Das Kapitel endet mit einer kurzen zusammenfassenden Darstellung des dynamisch-systemischen in Gegenüberstellung zum klassischen Weltbild.

Im vierten Kapitel werden die Ausführungen zu den eingangs erwähnten vier zentralen Prozessebenen vertieft. Die interpersonelle, die psychische, die gesellschaftlich-kulturelle und die körperliche Prozessebene werden fundiert und anschaulich dargestellt und in ihrem gleichzeitigen Zusammenwirken erläutert. Dies ist mit Exkursen zu Kommunikationstheorie, Gedächtnis, gemeinsamer Kreation von Sinn, und erneut Gestalttheorie sehr spannend zu lesen.

Das füntte Kapitel ist der Welt des Bewusstseins gewidmet. Es enthält eine Besinnung darauf, was mit «Person» gemeint ist. Zentral ist die Aussage, dass «Person» nicht angeboren ist, wohl aber das evolutionär vorstrukturierte Potenzial dazu. Damit sich jemand zur «Person» entfalten kann, braucht es hinreichend guter Entwicklungsbedingungen. Das psychosoziale Beziehungsangebot kann durch drei Kernaspekte einer Grundhaltung beschrieben werden, wie Carl Rogers diese in seinem personzentrierten
Ansatz beschrieben hat: nicht an Bedingungen geknüpfte Wertschätzung des Menschen als Subjekt, kongruentes Angebot von organismischen Erfahrungsmöglichkeiten und deren Symbolisierung, insbesondere durch sprachliche Begleitung, und schliesslich empathisches Einfühlen in die Bedürfnisse des anderen. "Der humanistische Kernsatz: «Nur am Du kann man zum Ich werden` erhält in der Personzentrierten Systemtheorie somit einen umfassenderen Kontext, der u. a. makro- und mikrosoziale, historische oder evolutionäre Perspektiven auf die aktuelle Du-Ich-Begegnung miteinbezieht» (S. 234).

Das sechste Kapitel trägt den Titel «Personzentrierte Systemtheorie im Kontext der Praxis». «Das Anliegen der Personzentrierten Systemtheorie ist es, das grosse Spektrum an vorhandenen Konzepten und Vorgehensweisen in Psychotherapie, Beratung und Coaching besser nutzen zu können» (S. 235). Der Autor erläutert, mit welchen Problemen man eher PsychotherapeutInnen, BeraterInnen oder Coaches bzw. Couchinnen aufsucht. Die professionelle Rolle umfasst als Hauptaufgabe die Begleitung bei «Ordnungs-Ordnungs-Übergängen». Damit sind Transformationen von Ordnungsstrukturen gemeint. Wichtig ist der Gebrauch von Intuition, Imagination und Kreativität.

Das Buch besticht dadurch, dass es nicht Partei nimmt für eine bestimmte psychotherapeutische Richtung, sondern mit der personzentrierten systemischen Sichtweise Bezüge zu vielen «Schulen» herstellt, um zu zeigen, was dort gemacht wird. Kriz beachtet den humanistischen Ethos, dem anderen mit Wertschätzung zu begegnen, wenn er auf andere Therapieansätze eingeht. Wiederholt kritisiert er aber zu Recht den aktuellen Trend zu manualisierten Therapien. Das entspräche nicht dem Bild einer dynamischen, systemischen und prozessorientierten Psychotherapiekonzeption

Subjekt und Lebenswelt behandelt viele grundlegende Themen aus Psychotherapie, Beratung und Coaching fundiert und ist reichlich mit Praxisbeispielen illustriert. In diesen Feldern tätige Menschen finden hier vertiefte Überlegungen und Anregungen zu ihrer Praxistätigkeit und zu deren theoretischen Verankerungen, egal zu welcher Therapierichtung sie sich zählen. Ich kann es gern empfehlen.

Peter Schulthess

\section{Hermann Wegscheider (2020). Dialog und Intersubjektivität in der Gestalttherapie. Von der jüdischen Tradition und Dialogphilosophie zu relationalen Entwicklungen in der Psychoanalyse und Gestalttherapie Gevelsberg: EHP, Verlag Andreas Kohlhage.} ISBN: 978-3-8979-7118-9. 248 S., 26,99 EUR, 37,70 CHF

Psychotherapie-Wissenschaft 10 (2) 106-107 2020

www.psychotherapie-wissenschaft.info

CC BY-NC-ND

https://doi.org/10.30820/1664-9583-2020-2-106

In diesem Buch wird dargelegt, wie manche europäische Therapierichtungen direkt oder indirekt geprägt sind von 
der jüdischen Tradition, insbesondere der chassidischen Tradition und dem Philosophen Martin Buber mit seinem einen spirituellen Anteil beinhaltenden Dialog-Ansatz.

In einem ersten Teil wird die Bedeutung des jüdischen Hintergrundes für die Psychotherapie erörtert. Die Ursprünge des dialogischen Vorgehens (z. B. im Judentum, im Chassidismus, in der Dialogphilosophie) und die Rezeption dialogischer Vorgehensweisen für die Praxis der Psychotherapie werden dargelegt. Jüdische Wurzeln haben Freud, Adler, Lewin, Moreno, Fromm, Perls, Frankl. Ob sie sich nun direkt auf diese Wurzeln berufen in ihren Therapieansätzen und ob sie religiös aktiv waren oder nicht, der Autor macht geltend, dass es einen indirekten biografischen Einfluss allein aufgrund der kulturellen Sozialisation gegeben haben muss. Eine Verbindung zwischen jüdischen religiösen und philosophischen Praktiken zur Psychotherapie wird gezogen, die von diesen beeinflusst sei. Die in vielen heutigen Psychotherapiekonzeptionen vertretene holistische Auffassung der Einheit von Körper, Psyche und Geist wird als Übernahme dieses jüdischen Verständnisses betrachtet. Das Kapitel enthält viele wissenswerte Darstellungen über den Gehalt und die Verbreitung des Chassidismus in Europa. Fromm soll im Chassidismus eine revolutionäre Massenbewegung gesehen haben, die einen humanistischen Sozialismus vertrat.

Im nächsten Teil des Buches fokussiert der Autor auf Bubers Dialogphilosophie und deren Verbindungen zu den Dialogphilosophien seiner Zeit, um in einem nächsten Kapitel die Entwicklung von der Dialogphilosophie zur dialogischen Psychotherapie zu beschreiben, als Folge dessen, dass die PsychotherapeutInnen und PsychologInnen die Dialogphilosophie entdeckt hätten. Genannt werden hier Adler, Binswanger, Kohut, Rollo May, Friedmann, Ferenzci, Balint, Sullivan, Jung, Rogers, Ruth Cohn und verschiedene GestalttherapeutInnen wie Rich Hycner, Lynne Jacobs und Gary Yontef. Sie hätten die dialogische Wende in der Psychotherapie eingeleitet, die Orientierung an der psychotherapeutischen Beziehungserfahrung, und die monologische Psychotherapie beendet.

Im nächsten Kapitel widmet sich der Autor dem dialogischen Ansatz in der klassischen Gestalttherapie. Dieser sei dort schon in den 1950er Jahren enthalten gewesen, später aber durch die im folgenden Kapitel erläuterten vier Phasen der dialogischen Entwicklung in der Gestalttherapie vertieft und klarer herausgearbeitet worden. $\mathrm{Zu}$ dieser dialogischen Wende in vier «Wellen»: Die erste Welle betont als theoretisches Merkmal das «Organismus-Umwelt-Feld», die zweite die Implizierung der Buber'schen «Ich-Du»-Beziehung in den psychotherapeutischen Kontext, die dritte die Intersubjektivitätstheorie und die vierte den «relationalen Grund». Hierbei wird ein dialogisches «Zwischen», ein «intersubjektives Drittes» postuliert. Mit Buber ist da auch die Anwesenheit des Göttlichen in der zwischenmenschlichen Begegnung gemeint, was sowohl der relationalen Psychoanalyse wie auch der Gestalttherapie etwas Transpersonales und einen religiösen Aspekt verleiht. Das dürfte sowohl in der rela- tionalen Psychoanalyse wie auch in der Gestalttherapie nicht widerspruchslos aufgenommen werden.

In einem weiteren Kapitel werden Verbindungen des relationalen Paradigmas in der Psychoanalyse und ihre Auswirkungen auf die Gestalttherapie diskutiert. Durch das relationale Paradigma sieht der Autor eine Annäherung von Psychoanalyse und Gestalttherapie, die schon mit der Emigration des Ehepaars Perls in die USA und die bereitwillige Aufnahme in der interpersonalen psychoanalytischen «Washington School of Psychiatry» ihren Ursprung nahm. Manchmal hatte ich bei meiner Lektüre den Eindruck, es sei dem Autor wichtig, dass die Gestalttherapie sich an diese neue Form der Psychoanalyse anlehnen kann. Es könnte ja auch umgekehrt sein, bzw. hoffentlich sehen sich beide Schulen als selbstständig, aber in einem schulenübergreifenden, gegenseitig befruchtenden Dialog.

Zum Schluss zieht der Autor wieder den Bogen zur jüdischen Denktradition und «der Bedeutung unmittelbarer Begegnung von Mensch zu Gott und von Mensch zu Mensch»(S. 203). Er erwähnt hier nicht nur erneut Buber, sondern geht auch auf Merleau-Ponty, Gadamer und Lévinas ein.

In einer Zusammenfassung stellt Wegscheider dar, wie er sieben Jahre an diesem Buch gearbeitet hat. Er arbeitet einige Essentials heraus und skizziert Ideen für künftige Forschungsprojekte.

Das Buch ist interessant zu lesen und gibt eine Fülle von Informationen über die jüdische Denktradition und deren impliziten oder expliziten Einfluss auf Therapieverfahren, die von jüdischen Psychotherapeuten initiiert wurden.

Was inhaltlich fehlt, ist bei der Beschreibung der relationalen Wende eine Reflexion zur zeitgeschichtlichen Entwicklung des letzten und dieses Jahrhunderts. Psychotherapie-Konzeptionen entstehen nicht im luftleeren Raum, sondern immer in einem kulturellen und zeitgeschichtlichen Kontext. Deren Veränderungen auch. Gerade wer feldtheoretisch denkt, sollte dies nicht ausser Acht lassen. Unsere Zeit der sozialen Isolierung grosser Teile der Bevölkerung, nicht zuletzt unter dem Einfluss der IT-Entwicklung, hat neue Störungsbilder erzeugt, die eine Beziehungsorientierung mit einer tragfähigen menschlichen Grundlage in allen Therapieansätzen verlangt. Es fehlen auch Hinweise auf die wellenartige Entwicklung in der Verhaltenstherapie, die heute ebenfalls viel dialogischer daherkommt als in ihrer Gründungszeit und die der therapeutischen Beziehung deutlich höhere Bedeutung schenkt als früher.

$\mathrm{Zu}$ guter Letzt hätte ich dem Autor und seinem Text ein besseres, strengeres Lektorat gewünscht. Das Buch enthält viele Redundanzen, die man hätte vermeiden können. In verschiedenen Kapiteln liest man die fast identischen Sätze, die man früher schon gelesen hat. Das ist wohl der Tribut, wenn man sieben Jahre an einem Text gearbeitet hat und zwei Beiträge, die schon woanders publiziert waren, einfügt.

Dennoch: Das Buch ist lesenswert!

Peter Schulthess 